\title{
Práticas integrativas e complementares: uma estratégia na promoção da saúde da mulher
}

\author{
Integrative and complementary practices: a strategy for the Promotion of women's health \\ Prácticas integradoras y complementarias: una estrategia para promover la salud de la mujer
}

Recebido: 12/07/2021 | Revisado: 26/07/2021 | Aceito: 14/12/2021 | Publicado: 21/12/2021

\author{
Larissa Kerlly Costa Pinheiro \\ ORCID: https://orcid.org/0000-0002-7253-4111 \\ Universidade Ceuma, Brasil \\ E-mail:larissapho@hotmail.com \\ Tânia Beatriz Gaspar De Souza \\ ORCID: https://orcid.org/0000-0002-3215-1861 \\ Universidade Ceuma, Brasil \\ E-mail:tbzgaspar@gmail.com \\ Nailde Melo Santos \\ ORCID: https://orcid.org/0000-0002-7109-5671 \\ Universidade Ceuma, Brasil \\ E-mail:naildems@terra.com.br \\ Ana Patricia Fonseca Coelho Galvão \\ ORCID: https://orcid.org/0000-0003-3376-5678 \\ Faculdade de Ciências Médicas da Santa Casa de São Paulo, Brasil \\ E-mail: apfcoelho@gmail.com \\ Marenilde Alves de Souza Melo \\ ORCID: https://orcid.org/0000-0002-0708-0109 \\ Universidade Ceuma, Brasil \\ E-mail:marenilde_melo@hotmail.com \\ Caroline Valicheli de Matos Martinelli \\ ORCID: https://orcid.org/0000-0002-3031-3193 \\ Universidade Ceuma, Brasil \\ E-mail:caroline.valichellimm@gmail.com \\ Cláudia Regina Silva dos Santos Cunha \\ ORCID: https://orcid.org/0000-0002-2859-3587 \\ Universidade Ceuma, Brasil \\ E-mail:claudia.cunha@ceuma.com.br \\ Laurenne Milhomem Sousa Moraes \\ ORCID: https://orcid.org/0000-0002-1658-398X \\ Universidade Ceuma, Brasil \\ E-mail: laurenne.milhomem@gmail.com \\ Ana Manuella Azevedo da Silva \\ ORCID: https://orcid.org/0000-0002-9428-2445 \\ Universidade Federal do Piauí, Brasil \\ E-mail: ramanuelaazevedo@hotmail.com \\ Julieta Carvalho Rocha \\ ORCID: https://orcid.org/0000-0002-9980-4839 \\ Universidade Ceuma, Brasil \\ E-mail:julietha_rocha@hotmail.com \\ Joelmara Furtado dos Santos Pereira \\ ORCID: https://orcid.org/0000-0002-4220-4437 \\ Universidade Federal do Maranhão, Brasil \\ E-mail:joelmara29@gmail.com \\ Francisca Bruna Arruda Aragão \\ ORCID: https://orcid.org/0000-0002-1191-0988 \\ Universidade de São Paulo, Brasil \\ E-mail:aragao_bruna@usp.br
}

\section{Resumo}

Por possuir especificidades decorrentes de sua condição de gênero e de sexo, a população feminina demanda uma assistência cada vez menos intervencionista, medicamentosa e centrada na doença. Portanto, o objetivo deste estudo foi analisar a utilização das Práticas Integrativas e Complementares (PIC) como estratégia de promoção da Saúde da Mulher, verificando as PICs mais utilizadas, o contexto da vida da mulher em que são aplicadas e quais benefícios elas trazem a esse público. Este estudo configura-se como uma revisão integrativa da literatura desenvolvida nas seguintes bases de dados: LILACS, MEDLINE e BDENF, utilizando operadores booleanos combinados com os 
descritores da saúde a partir do DeCS nas seguintes combinações: "Terapias Complementares" AND Saúde da Mulher"; "Medicina Integrativa" AND "Saúde da Mulher"; "Medicina Tradicional” AND "Saúde da Mulher" e pelo nome de cada PIC existente no SUS atualmente AND "Saúde da Mulher". Aamostra final foi composta por 16 artigos, sendo 10 da MEDLINE e 6 da LILACS. Os estudos abordavam a utilização de terapias como musicoterapia, acupuntura e suas técnicas, ioga, relaxamento guiado, hidroterapia, técnicas de respiração, entre outras. As PICs foram aplicadas em mulheres experienciando gestação, trabalho de parto, SOP, trabalho, tabagismo, câncer, climatério e terceira idade. As terapias alternativas se mostraram uma opção viável no tratamento e na promoção da saúde atuando de forma complementar à medicina convencional.

Palavras-chave: Medicina tradicional; Medicina integrativa; Saúde da mulher; Terapias complementares.

\begin{abstract}
For having specificities arising from their gender and sex condition, the female population demands an assistance that is less and less interventionist, medicated, and disease-centered. Therefore, the objective of this study was to analyze the use of Integrative and Complementary Practices (ICPs) as a strategy to promote Women's Health, verifying the most used PICs, the context of women's lives in which they are applied, and what benefits they bring to this public. This study is an integrative literature review developed in the following databases: LILACS, MEDLINE and BDENF, using Boolean operators combined with health descriptors from DeCS in the following combinations: "Complementary Therapies" AND "Women's Health"; "Integrative Medicine" AND "Women's Health"; "Traditional Medicine" AND "Women's Health" and by the name of each PIC currently in SUS AND "Women's Health". The final sample was composed of 16 articles, 10 from MEDLINE and 6 from LILACS. The studies addressed the use of therapies such as music therapy, acupuncture and its techniques, yoga, guided relaxation, hydrotherapy, and breathing techniques, among others. The PICs were applied to women experiencing pregnancy, labor, SOP, work, smoking, cancer, climacteric and elderly. Alternative therapies proved to be a viable option in the treatment and promotion of health, acting as a complement to conventional medicine.
\end{abstract}

Keywords: Medicine traditional; Integrative medicine; Women's health; Complementary therapies.

\title{
Resumen
}

Por sus especificidades derivadas de su género y condición de género, la población femenina demanda una atención cada vez menos intervencionista, farmacológica y centrada en la enfermedad. Por tanto, el objetivo de este estudio fue analizar el uso de Prácticas Integrativas y Complementarias (PIC) como estrategia de promoción de la Salud de la Mujer, verificando los PIC más utilizados, el contexto de vida de las mujeres en el que se aplican y qué beneficios aportan a la salud. este público. Este estudio es una revisión integradora de la literatura desarrollada en las siguientes bases de datos: LILACS, MEDLINE y BDENF, utilizando operadores booleanos combinados con descriptores de salud de DeCS en las siguientes combinaciones: "Terapias complementarias" Y "Salud de la mujer"; "Medicina Integrativa" Y "Salud de la Mujer"; "Medicina Tradicional" Y "Salud de la Mujer" y por el nombre de cada PIC existente en el SUS actualmente Y "Salud de la Mujer". La muestra final estuvo formada por 16 artículos, 10 de MEDLINE y 6 de LILACS. Los estudios abordaron el uso de terapias como la musicoterapia, la acupuntura y sus técnicas, yoga, relajación guiada, hidroterapia, técnicas de respiración, entre otras. Los PIC se aplicaron a mujeres que experimentan embarazo, trabajo de parto, síndrome de ovario poliquístico, trabajo de parto, tabaquismo, cáncer, climaterio y vejez. Las terapias alternativas demostraron ser una opción viable en el tratamiento y promoción de la salud, actuando de forma complementaria a la medicina convencional.

Palabras clave: Medicina tradicional; Medicina integrativa; Salud de la mujer; Terapias complementarias.

\section{Introdução}

Segundo a Organização Mundial da Saúde (2013), a Medicina Tradicionale Complementar (MTC), um conjunto de práticas e saberes que perpassam gerações de diferentes culturas e povos e que não fazem parte do arsenal terapêutico da medicina convencional de um país, é constituída pelas Práticas Integrativas e Complementares (PICs), que tratam o indivíduo em sua integralidade e contribuem de forma interdisciplinar no cuidado à saúde. Com base em evidências científicas acerca de sua eficácia, as PICs são práticas terapêuticas que estão sendo gradualmente incorporadas ao rol de procedimentos da medicina ocidental (Telesi Junior, 2016).

Nesta perspectiva, o panorama global desenhado pela Coordenação Nacional de Práticas Integrativas e Complementares em Saúde (2020) mostra que a adesão dessas terapias vem acontecendo em larga escala nos continentes da América do Norte, África e Europa.

Já no contexto nacional, a demanda por novas práticas de assistência à saúde emergiu no Brasil com a realização da VIII Conferência Nacional de Saúde, em 1986 (Motta \& Marchiori, 2013). Entretanto, somente em 2006, com a apresentação 
da portaria GM/MS n 971, foi aprovada a Política Nacional de Práticas Integrativas e Complementares (PNPIC) no Sistema Único de Saúde (SUS),que surgiu com o objetivo de atender à necessidade de ampliação e melhoria das terapêuticas oferecidas na rede pública de saúde, trazendo em seu texto a implementação de cinco práticas. Em 2017, foram incluídas catorze outras PICs e, em 2018, ampliou-se a PNPIC com a inclusão de dez novos recursos, condensando em vinte e nove práticas terapêuticas complementares existentes hoje no SUS (SES-MG, 2020).

Seguindo a tendência mundial e nacional, a Agência de Notícias do Governo do Estado do Maranhão (2019) publicou que 179 municípios do Estado já ofereciam atendimento de PICs no ano de 2018 em Unidades de Saúde da Família (USF). A oferta dessas terapias no sistema público de saúde se justifica pelos benefícios já comprovados quanto a sua ação holística (mente, corpo e espírito) no processo saúde-doença (Santos \& Santos, 2017).

Por possuir especificidades provenientes de sua condição de gênero e sexo, a população feminina demanda uma reflexão acerca da importância do uso de uma assistência cada vez menos intervencionista, medicamentosa e centrada na doença. Nesse âmbito, o presente estudo objetiva analisar a utilização das Práticas Integrativas e Complementares como estratégia de promoção da saúde da mulher à luz da literatura, verificando seus benefícios.

\section{Metodologia}

Esta revisão integrativa da literatura foi realizada seguindo um protocolo: delimitou-se a pergunta norteadora, foram definidas as bases de dados, bem como os critérios de inclusão e exclusão para a seleção dos artigos da amostra.

Este estudo pautou-se, pois, tendo em conta a estratégia PICO (Santos, et al., 2007), na seguinte questão norteadora: quais os efeitos da utilização das práticas integrativas e complementares na promoção da saúde da mulher?

Quadro 1 - Descrição da estratégia PICO. São Luís, MA, Brasil, 2021.

\begin{tabular}{|c|c|c|}
\hline Acrônimo & Definição & Descrição \\
\hline P & População & Mulheres \\
\hline I & Intervenção & Práticas integrativas \\
\hline C & Controle ou comparação & $\begin{array}{l}\text { Os efeitos da utilização das práticas integrativas e } \\
\text { complementares na promoção da saúde da mulher }\end{array}$ \\
\hline O & Desfechos ("outcomes") & Promoção na saúde da mulher \\
\hline
\end{tabular}

Fonte: Autores (2021).

A coleta de dados foi operada nas seguintes bases de dados: Base de Dados de Enfermagem (BDENF), Base de Dados Latino-Americana e do Caribe em Ciências da Saúde (LILACS) e Medical Literature Analysis and Retrieval Sistem on-line (MEDLINE), realizada no primeiro semestre de 2021.

Para a seleção de artigos, foram definidos os seguintes critérios de inclusão: i) artigos publicados nos idiomas português, espanhol e inglês; ii) artigos que abordassem a temática referente à pesquisa e publicados dentro do recorte temporal dos últimos cinco anos. Os critérios de exclusão adotados foram: artigos de revisão integrativa ou sistemática, relatórios, dissertações de mestrado, teses de doutorado, entrevistas e artigos que abordassem utilização das PICs em outros grupos.

A pesquisa consistiu em três etapas. A primeira se deu pela aplicação da ferramenta de busca avançada nas bases de dados, com base nos descritores da saúde, que foram definidos a partir do DeCS. Foram pesquisadas suas combinaçõescom o operador booleano AND da seguinte forma: "Terapias Complementares" AND "Saúde da Mulher"; "Medicina Integrativa" AND "Saúde da Mulher"; e "Medicina Tradicional" AND "Saúde da Mulher". Além disso, pesquisou-se por nome de cada uma das práticas integrativas e complementares existentes hoje no SUS combinados com a expressão booleana AND e o termo "Saúde da Mulher" e, pela leitura dos títulos dos artigos disponíveis, aplicou-se o primeiro filtro de exclusão: estudos 
com títulos que não abordavam o assunto de interesse da pesquisa. $\mathrm{Na}$ segunda etapa, dois pesquisadores independentes realizaram a leitura criteriosa dos resumos de cada artigo coletado nas bases de dados e cujo julgamento ocorreu conforme os critérios de inclusão e exclusão, foram excluídos também os artigos repetidos. Por fim, a terceira etapa consistiu na leitura na íntegra de cada artigo selecionado na etapa anterior. Nos casos em que houve dúvida ou discrepância entre os pesquisadores, um terceiro pesquisador foi consultado.

Os dados extraídos dos artigos selecionados foram transcritos para um instrumento validado, que foi adaptado para atender ao objetivo do estudo (Ursi \& Galvão, 2006). O instrumento contém variáveis de interesse da pesquisa, cujos itens são: autores, periódico em que foram publicados, ano de publicação, país do estudo, delineamento do estudo, nível de evidência e principais resultados. Adotou-se a proposta descrita por Melnyk e Fineout-Overholt (2005) para analisar o delineamento de pesquisa e classificar o nível das evidências científicas dos artigos.

\section{Resultados}

Na primeira etapa desta pesquisa, após utilizar as combinações de descritores e PICs e leitura dos títulos, obtivemos um total de 181 (cento e oitenta e um) artigos coletados. Da segunda etapa, após a leitura dos resumos e sendo aplicados os critérios de inclusão e exclusão, restaram 29 (vinte e nove) artigos e, destes, foram excluídos mais cinco artigos, uma vez que se tratavam de artigos duplicados, resultando 24 (vinte e quatro) estudos para a etapa seguinte. Na terceiraetapa, após a leitura na íntegra, foram excluídos mais oito artigos. Assim, a amostra final ficou constituída por 16 (dezesseis) artigos, sendo 0 (zero) na BDENF, 6 (seis) na LILACS e 10 (dez) na MEDLINE.

Figura 1 - Fluxograma de etapas da coleta e seleção dos artigos que integraram este estudo.

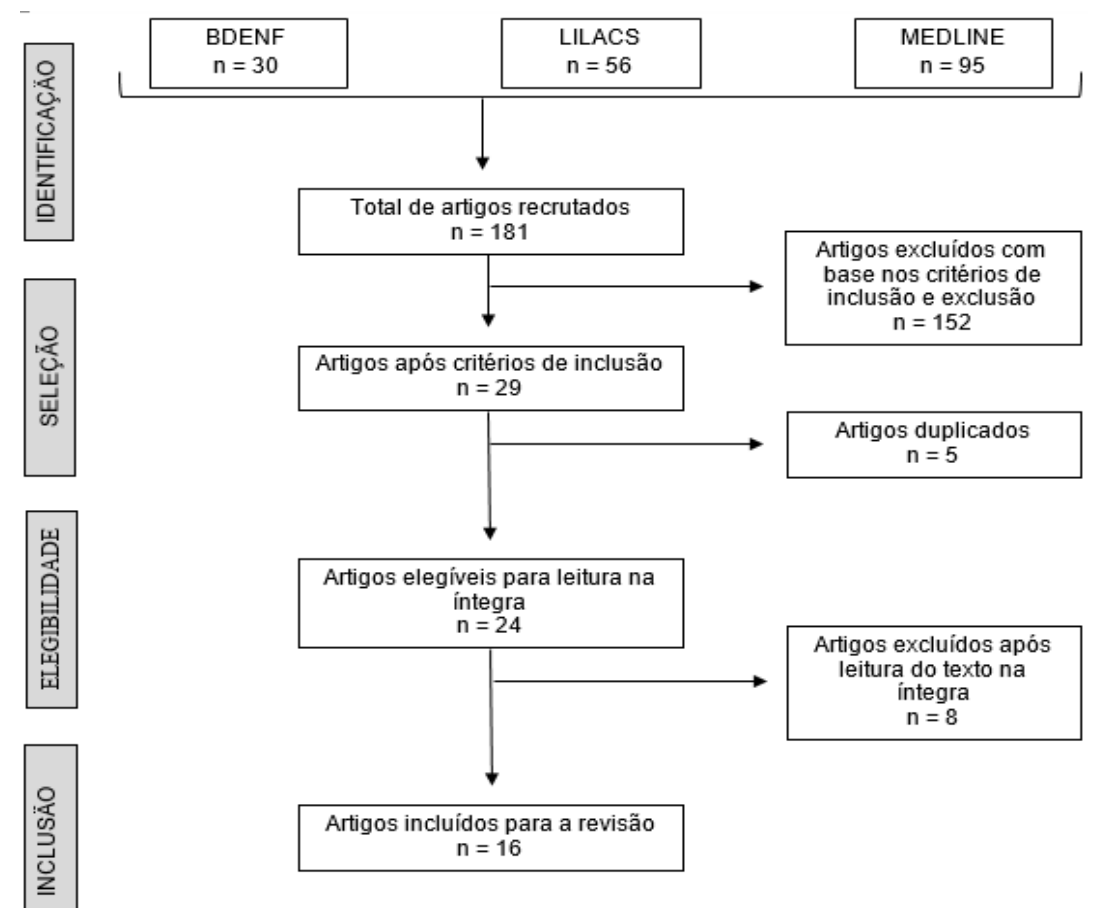

Fonte: Autores (2021).

Os 16 (dezesseis) artigos que integraram a amostra deste estudo estãodispostos no quadro 2, identificados pelas variáveis de interesse da pesquisa. 
Quadro 2 - Artigos integrantes da amostra.

\begin{tabular}{|c|c|c|c|c|c|}
\hline & Autores & Periódico & Ano/país & $\begin{array}{l}\text { Desenho do } \\
\text { estudo/ nível } \\
\text { de evidência }\end{array}$ & Principais resultados \\
\hline 1 & $\begin{array}{l}\text { Buglione, A } \\
\text { et al. }\end{array}$ & $\begin{array}{c}\text { Archives of } \\
\text { Gynecology } \\
\text { and Obstetrics }\end{array}$ & $\begin{array}{l}\text { 2020/ } \\
\text { Itália }\end{array}$ & $\begin{array}{l}\text { Ensaio } \\
\text { clínico } \\
\text { randomizado/ } \\
\text { nível II }\end{array}$ & $\begin{array}{l}\text { Músicoterapia no trabalho de parto: } \\
\text { - diminuição da dor durante a fase ativa do trabalho de parto. } \\
\text { - diminuição da dor em uma hora pós- parto. } \\
\text { - diminuição da ansiedade durante a fase ativa do trabalho de } \\
\text { parto, segundoestágio do parto e uma hora após o } \\
\text { parto. }\end{array}$ \\
\hline 2 & $\begin{array}{l}\text { Wang, Zet } \\
\text { al. }\end{array}$ & $\begin{array}{l}\text { Acupuncture } \\
\text { in Medicine }\end{array}$ & $\begin{array}{l}2019 / \\
\text { China }\end{array}$ & $\begin{array}{l}\text { Ensaio } \\
\text { clínico } \\
\text { randomizado } \\
\text { controlado/ } \\
\text { nível II }\end{array}$ & $\begin{array}{l}\text { Eletroacupuntura em mulheres com Síndrome dos Ovários } \\
\text { Policísticos (SOP): } \\
\text { diminuição dos escores das escalas de ansiedade e depressão; } \\
\text { aumento dos escores de diversos domínios do SF-36, dos escores } \\
\text { totais da qualidade de vida chinesa e dos escores } \\
\text { da qualidade de vida da mulher com SOP (emoção, pelos } \\
\text { corporais e problemas de infertilidade). }\end{array}$ \\
\hline 3 & $\begin{array}{l}\text { Ozcan, } \mathrm{H} \\
\text { et al. }\end{array}$ & $\begin{array}{l}\text { African } \\
\text { Health } \\
\text { Sciences }\end{array}$ & $\begin{array}{c}\text { 2019/ } \\
\text { Turquia }\end{array}$ & $\begin{array}{l}\text { Estudo } \\
\text { transversal/ } \\
\text { nível VI }\end{array}$ & $\begin{array}{l}\text { Fitoterapia, suplementos alimentares, religiosidade, práticas } \\
\text { mente-corpo na menopausa: } \\
\text { eficácia no tratamento das ondas de calor causadas pela } \\
\text { menopausa. }\end{array}$ \\
\hline 4 & $\begin{array}{l}\text { Cicek, S; } \\
\text { Basar, F. }\end{array}$ & $\begin{array}{l}\text { Complement } \\
\text { ary Therapies } \\
\text { inClnical } \\
\text { Practice }\end{array}$ & $\begin{array}{c}2017 / \\
\text { Turquia }\end{array}$ & $\begin{array}{l}\text { Estudo } \\
\text { randomizado } \\
\text { controlado/ } \\
\text { nível II }\end{array}$ & $\begin{array}{l}\text { Técnicas de respiração no trabalho departo: } \\
\text { - melhora do escore de ansiedade na fase ativa tardia do } \\
\text { trabalho de parto; } \\
\text { - diminuição da duração média nas fases latente e ativa do } \\
\text { trabalho de parto. }\end{array}$ \\
\hline 5 & $\begin{array}{l}\text { Fancourt, } \\
\quad \text { D; } \\
\text { Perkins, R. }\end{array}$ & BMJ Open & $\begin{array}{c}2018 / \\
\text { Inglaterra }\end{array}$ & $\begin{array}{l}\text { Estudo de } \\
\text { coorte } \\
\text { prospectivo/ } \\
\text { nível IV }\end{array}$ & $\begin{array}{l}\text { Musicoterapia na gestação: } \\
\text { • elevação dos níveis de bem-estar; } \\
\text { • redução dos sintomas de depressão pós-parto nos } 3 \text { primeiros } \\
\text { meses após o nascimento. }\end{array}$ \\
\hline 6 & Tsai, SY. & $\begin{array}{l}\text { International } \\
\text { Journal of } \\
\text { Environment } \\
\text { al Research } \\
\text { and Public } \\
\text { Health }\end{array}$ & $\begin{array}{c}2016 / \\
\text { Taiwan }\end{array}$ & $\begin{array}{l}\text { Estudo de } \\
\text { intervenção/ } \\
\text { nível II }\end{array}$ & $\begin{array}{l}\text { Ioga em mulheres com síndrome pré-menstrual: } \\
\text { - diminuição dos efeitos moderados ou graves da dor } \\
\text { menstrual no trabalho; } \\
\text { - diminuição do uso de analgésicos durantea menstruação. } \\
\text { - melhora dos escores do SF (função físicae dor corporal); } \\
\text { - diminuição do inchaço abdominal, sensibilidade } \\
\text { mamária, } \\
\text { abdominais e suores frios; }\end{array}$ \\
\hline 7 & $\begin{array}{l}\text { Kao, YHet } \\
\text { al. }\end{array}$ & $\begin{array}{l}\text { Journal of } \\
\text { Alternative } \\
\text { and } \\
\text { Complement } \\
\text { ary Medicine }\end{array}$ & $\begin{array}{c}2017 / \\
\text { Taiwan }\end{array}$ & $\begin{array}{l}\text { Ensaio } \\
\text { clínico } \\
\text { randomizado } \\
\text { controlado/ } \\
\text { nível II } \\
\end{array}$ & $\begin{array}{l}\text { Aromaterapia e massagem de acupressão em mulheres de } \\
\text { carreira: } \\
\text { - melhoria na qualidade do sono; } \\
\text { - melhoria de qualidade de vida; }\end{array}$ \\
\hline 8 & $\begin{array}{l}\text { Kashani,L } \\
\quad \text { et al. }\end{array}$ & $\begin{array}{l}\text { Archives of } \\
\text { Gynecology } \\
\text { and Obstetrics }\end{array}$ & $\begin{array}{l}\text { 2018/ } \\
\text { Irã }\end{array}$ & $\begin{array}{l}\text { Ensaio duplo- } \\
\text { cego } \\
\text { randomizado } \\
\text { controlado/ } \\
\text { nível II } \\
\end{array}$ & $\begin{array}{l}\text { Fitoterapia (açafrão) em mulheres pós- menopausa: } \\
\text { - redução dos escores da Escala de Interferência Diária } \\
\text { Relacionada às Ondas de Calor; } \\
\text { - redução dos escores da Escala de Avaliação de Depressão } \\
\text { Hamilton. }\end{array}$ \\
\hline 9 & $\begin{array}{l}\text { Kirshbau } \\
\text { m, MN; } \\
\text { Stead, M; } \\
\text { Bartys, S. }\end{array}$ & $\begin{array}{l}\text { International } \\
\text { Journal of } \\
\text { Palliative } \\
\text { Nursing }\end{array}$ & $\begin{array}{c}2016 / \\
\text { Austrália }\end{array}$ & $\begin{array}{l}\text { Estudo } \\
\text { transversal } \\
\text { qualitativo/ } \\
\text { nível VI }\end{array}$ & $\begin{array}{l}\text { Reiki em mulheres com câncer: } \\
\text { • melhora na liberação de tensão emocional e sensações físicas } \\
\text { durante assessões; } \\
\text { • benefícios físicos, emocionais e cognitivos após a terapia. }\end{array}$ \\
\hline 10 & $\begin{array}{l}\text { Rosen, RK } \\
\quad \text { et al. }\end{array}$ & $\begin{array}{l}\text { International } \\
\text { Journal of } \\
\text { Yoga Therapy }\end{array}$ & $\begin{array}{l}\text { 2016/ } \\
\text { Estados } \\
\text { Unidos }\end{array}$ & $\begin{array}{l}\text { Ensaio } \\
\text { clínico } \\
\text { randomizado } \\
\text { controlado/ } \\
\text { nível II }\end{array}$ & $\begin{array}{l}\text { Ioga para mulheres que fumam: } \\
\text { • melhora da respiração; } \\
\text { - ampliação da consciência do corpo e darespiração; } \\
\text { aumento da sensação de bem-estar. }\end{array}$ \\
\hline 11 & $\begin{array}{l}\text { Fernández, } \\
\text { FXA; } \\
\text { Pino- } \\
\text { Juste, M; } \\
\text { Pérez, } \\
\text { JJN. }\end{array}$ & $\begin{array}{c}\text { Saúde e } \\
\text { Sociedade }\end{array}$ & $\begin{array}{c}2020 / \\
\text { Espanha }\end{array}$ & $\begin{array}{l}\text { Estudo } \\
\text { etnográfico } \\
\text { descritivo/ } \\
\text { nível VI }\end{array}$ & $\begin{array}{l}\text { Programa grupal de tratamento nãofarmacológico para mulheres } \\
\text { com câncer: } \\
\text { • melhora no enfrentamento da doença e efeitos colaterais do } \\
\text { tratamento,aumentando o bem-estar e segurança; } \\
\text { melhoria das habilidades de relacionamento social. }\end{array}$ \\
\hline 12 & $\begin{array}{l}\text { Rodriguez, } \\
\text { MPC etal. }\end{array}$ & $\begin{array}{l}\text { Investigación } \\
\text { en } \\
\text { Enfermería: }\end{array}$ & $\begin{array}{c}2018 / \\
\text { Portugal }\end{array}$ & $\begin{array}{l}\text { Estudo } \\
\text { qualitativo/ } \\
\text { nível VI }\end{array}$ & $\begin{array}{l}\text { Análise bioenergética em mulheres idosas: } \\
\text { aumento do bem-estar, vitalidade } \\
\text { consciência corporal. }\end{array}$ \\
\hline
\end{tabular}


Research, Society and Development, v. 10, n. 17, e87101718147, 2021

(CC BY 4.0) | ISSN 2525-3409 | DOI: http://dx.doi.org/10.33448/rsd-v10i17.18147

\begin{tabular}{|c|c|c|c|c|c|}
\hline & Autores & Periódico & Ano/país & $\begin{array}{l}\text { Desenho do } \\
\text { estudo/ nível } \\
\text { de evidência }\end{array}$ & Principais resultados \\
\hline & & $\begin{array}{l}\text { Imagen y } \\
\text { Desarrollo }\end{array}$ & & & \\
\hline 13 & $\begin{array}{l}\text { Martins, } \\
\text { ES et al. }\end{array}$ & $\begin{array}{l}\text { Revista de } \\
\text { Pesquisa } \\
\text { (Universidad e } \\
\text { Federal do } \\
\text { Estado do Rio } \\
\text { de Janeiro } \\
\text { Online) }\end{array}$ & $\begin{array}{l}2020 / \\
\text { Brasil }\end{array}$ & $\begin{array}{l}\text { Estudo } \\
\text { transversal/ } \\
\text { nível VI }\end{array}$ & $\begin{array}{l}\text { Acupuntura na gestação: } \\
\text { - benefícios físicos (maior relaxamento do corpo; aumento de } \\
\text { energia e disposição; melhoria do sono, dores na cabeça, pernas, } \\
\text { câimbras, funcionamento intestinal, edema nas pernas, dor } \\
\text { lombar e outras dores); } \\
\text { - benefícios emocionais (melhora do humor, estresse, paciência } \\
\text { e ansiedade). } \\
\text { - outros benefícios (aumento da autoconfiança, autoestima, } \\
\text { ânimo, autocontrole, flexibilidade e melhora de } \\
\text { dores nas articulacões). }\end{array}$ \\
\hline 14 & Silva, RM & $\begin{array}{c}\text { Saúde e } \\
\text { Sociedade }\end{array}$ & $\begin{array}{l}\text { 2016/ } \\
\text { Brasil }\end{array}$ & $\begin{array}{l}\text { Estudo } \\
\text { qualitativo/ } \\
\text { nível VI }\end{array}$ & $\begin{array}{l}\text { Fitoterapia, reiki, florais, homeopatia, shiatsu, acupuntura, } \\
\text { hidroterapia, massagem terapêutica, meditação, visualização, } \\
\text { relaxamento, técnicas de respiração, ioga, moxabustão na } \\
\text { gestação: } \\
\text { • diminuição do tempo do trabalho de parto, encaixe do bebê, } \\
\text { indução natural do parto e melhor controle da dor; } \\
\text { - empoderamento da mulher no momento do parto. }\end{array}$ \\
\hline 15 & $\begin{array}{l}\text { Toneti, BF } \\
\text { et al. }\end{array}$ & $\begin{array}{l}\text { Revista da } \\
\text { Escola de } \\
\text { Enfermagem } \\
\text { da USP }\end{array}$ & $\begin{array}{l}\text { 2019/ } \\
\text { Brasil }\end{array}$ & $\begin{array}{l}\text { Estudo } \\
\text { qualitativo/ } \\
\text { nível VI }\end{array}$ & $\begin{array}{l}\text { Relaxamento guiado em mulheres comcâncer de mama: } \\
\text { - melhoria do bem-estar; } \\
\text { - sensação de leveza; } \\
\text { relaxamento físico e mental. }\end{array}$ \\
\hline 16 & $\begin{array}{l}\text { Martins, } \\
\text { ES et al. }\end{array}$ & $\begin{array}{l}\text { Revista da } \\
\text { Escola de } \\
\text { Enfermagem } \\
\text { da USP }\end{array}$ & $\begin{array}{l}\text { 2018/ } \\
\text { Brasil }\end{array}$ & $\begin{array}{l}\text { Estudo } \\
\text { quase- } \\
\text { experimental/ } \\
\text { nível III }\end{array}$ & $\begin{array}{l}\text { Acupuntura em gestantes: } \\
\text { - redução na dor lombar logo a partir dasegunda sessão e } \\
\text { diminuição gradativa } \\
\text { com o avançar do número de sessões. }\end{array}$ \\
\hline
\end{tabular}

Fonte: Autores (2021).

O Quadro 3 identifica as terapias alternativas utilizadas em cada estudo daamostra e o contexto de vida da mulher em que elas foram aplicadas. 
Quadro 3 - Terapias utilizadas nos artigos selecionados e contexto de vida da mulher em que foramaplicadas.

\begin{tabular}{|c|c|c|}
\hline & Terapia utilizada & Contexto de vida \\
\hline 1 & Musicoterapia & Trabalho de parto \\
\hline 2 & Eletroacupuntura & Síndrome do ovário policístico \\
\hline 3 & $\begin{array}{l}\text { Fitoterapia, suplementos alimentares, religiosidade,práticas } \\
\text { mente-corpo }\end{array}$ & Menopausa \\
\hline 4 & Técnicas de respiração & Trabalho de parto \\
\hline 5 & Musicoterapia & Gestação \\
\hline 6 & Ioga & Síndrome pré-menstrual \\
\hline 7 & Aromaterapia, massagem de acupressão & Mulheres de carreira \\
\hline 8 & Fitoterapia & Pós-menopausa \\
\hline 9 & Reiki & Câncer \\
\hline 10 & Ioga & Tabagismo \\
\hline 11 & Grupo interdisciplinar de tratamento não-farmacológico & Câncer \\
\hline 12 & Bioenergética & Terceira idade \\
\hline 13 & Acupuntura & Gestação \\
\hline 14 & $\begin{array}{l}\text { Fitoterapia, reiki, florais, homeopatia, shiatsu, acupuntura, } \\
\text { hidroterapia, massagem terapêutica, } \\
\text { meditação, visualização, relaxamento, técnicas derespiração, } \\
\text { ioga, moxabustão }\end{array}$ & Gestação \\
\hline 15 & Relaxamento guiado & Câncer de mama \\
\hline 16 & Acupuntura & Gestação \\
\hline
\end{tabular}

Fonte: Autores (2021).

\section{Discussão}

Os artigos selecionados abordam os efeitos de uma gama de terapias alternativas emolduradas na Medicina Tradicional e Complementar aplicadas ou recomendadas em diversas etapas da vida das mulheres participantes dos estudos.

Segundo Bocanegra et al. (2020), a experiência da gestação é marcante para toda mulher que a vivencia, o que é suficiente para justificar o uso de tratamentos não-convencionais em conjunto com a medicinaalopática, objetivando garantirlhes uma melhor vivência. Corroborando essa afirmação, a musicoterapia foi utilizada em dois estudos envolvendo mulheres durante a gravidez. Buglione et al. (2020) avaliaram a técnica em nulíparas com gravidez única a termo e parto espontâneo, o que resultou em menor nível de dor durante o trabalho de parto e, em uma hora após o parto, uma diminuição do nível de ansiedade durante o trabalho de parto, parto e uma hora de pós-parto. Fancourte Perkins (2018) utilizaram a música em gestantes no último trimestre da gravidez e verificaram significativa melhora nos níveis de bem-estar e a diminuição dos sintomas de depressão pós-parto nos três primeiros meses apóso nascimento.

Ainda sobre gestação e parto, Silva et al. (2016) mostraram que doulas recomendam uso de chás e ervas medicinais, acupuntura, reiki, homeopatia, florais, shiatsu às gestantes e fazem uso de hidroterapia, massagem terapêutica, meditação, visualização, relaxamento, técnicas de respiração, ioga e moxabustão em suas pacientes e observaram uma diminuição do tempo de trabalho de parto, melhor controle da dor, empoderamento e autonomia da mulher, ajuda na tomada dedecisões, singularidade, respeito, além da humanização do parto. Em consonância com esses resultados, Cicek e Basar (2017) concluíram que técnicas de respiração são eficazes para melhor controle de ansiedade e diminuição da duração do trabalho de parto.

As gestantes que integraram os estudos de Martins et al. $(2018,2020)$, ao receberem intervenções com acupuntura, relataram mudança positiva em diversos desconfortos físicos e emocionais presentes durante a gravidez, tais como lombalgias, não-relaxamento do corpo, perda de qualidade do sono, falta de energia, dores na cabeça, nas pernas, câimbras, mal funcionamento intestinal, edema nas pernas, e outras dores, além de mudanças no humor, estresse, paciência e ansiedade. Wang et al. (2019) utilizaram a eletroacupuntura em mulheres solteiras com Síndrome dos Ovários Policísticos (SOP) e seu estudo mostrou que a aplicaçãoda técnica é capaz de regular a secreção de neurotransmissores, e, portanto, evidenciou melhora 
da ansiedade e depressão nessas mulheres, e dos escores das escalas de qualidade de vida. Considerando os resultados positivos que a acupuntura apresenta em relação à assistência a gestantes, Martins et al. (2017) alertam para a necessidade de profissionais de saúde, em especial os envolvidos no pré-natal, estarem informados sobre tratamentos como a acupuntura nessas pacientes.

Com a gestação e parto, as mulheres, muitas vezes ficam submetidas à dupla jornada de trabalho. Considerando a grande exposição ao estresse, Kao et al. (2017) desenvolveram um estudo que evidencia que tanto a inalação de óleos essenciais, quanto a massagem de acupressão são eficazes na promoção da qualidade de vida e do sono e de mulheres dentro deste contexto.

Ainda sobre jornada de trabalho, Tsai (2016) verificou que 12 (doze) semanas de intervenção em uma empresa, utilizando ioga em funcionárias com síndrome pré-menstrual, contribuíram com a redução do uso de analgésicos pelas participantes durante a menstruação, pois promoveram diminuição das dores e sensibilidades relacionadas à Tensão PréMenstrual (TPM), além de terem sido eficientes na melhora da função física. Corroborando esses resultados, Rosen, et al. (2016) evidenciaram os benefícios da terapia com ioga para as participantes fumantes, que incluíram ampliação da consciência do corpo, a melhora da respiração e da sensação de bem-estar, além de ter sido uma facilitadora na resposta a situações estressantes e no controle do desejo de fumar. O fortalecimento físico, social e mental proporcionado pela ioga, justifica sua popularização no Ocidente, especialmente na saúde da mulher, que por sua complexidade, pode se valer de diversas benesses deste recurso (Styles et al., 2019).

Assim como o tabagismo pode ter início por exposição ao estresse, este pode integrar a relação multicausal de alguns tipos de câncer. Portanto, Kirshbaum et al. (2016), após entrevistas com mulheres com câncer e que receberam terapia de reiki, verificaram que a técnica proporciona melhora espiritual, física, psicológica, emocional e cognitiva, demonstrando que pode ser utilizada como complemento para o tratamento convencional.

Indo na mesma direção, ao analisarem edições de um programa grupal de tratamento não-farmacológico para mulheres no enfretamento do câncer, Fernández et al. (2020) perceberam que as participantes relataram satisfação com o grupo, benefícios na aceitação, aquisição de conhecimentos, manejo no enfrentamento à doença e melhora do bem-estar. Concordando com a utilização das PICs, Toneti et al. (2019) avaliaram mulheres experienciando câncer de mama, e, em seu estudo, utilizaram a terapia de relaxamento guiado. Foram constatados relatos de sensação de leveza, bem-estar e relaxamento físico e mental, além da facilitação na compreensão do processo, de forma que esta terapia pode ser uma estratégia terapêutica no enfrentamento e tratamento da doença.

Com o grande número de mulheres em idade não reprodutiva, Banazeski et al. (2021) pontuam que é imprescindível que as Políticas Nacionais de Saúde lancem um olhar mais atento às ações para mulheres vivenciando o climatério. Quanto ao tratamento das ondas de calor causadas pela menopausa, dois estudos avaliaram efeitos de PICs em mulheres que não apresentavam menstruação nos últimos 12 (doze) meses. Ozcan et al. (2019) destacam que o uso de remédios fitoterápicos, suplementos alimentares, prática religiosa e exercícios mente-corpo são métodos complementares e alternativos que se mostraram eficazes no tratamento das ondas de calor causadas pela menopausa. Enquanto Kashani et al. (2018) apontam para a segurança e eficácia do tratamento com cápsulas de açafrão,mostrando resultados positivos após seis semanas.

Passados alguns anos da menopausa, a mulher passa a experienciar a terceira idade e, visando a melhoria na qualidade de vida desta população, Rodriguez et al. (2018) viram na análise bioenergética, que relaciona o corpo e sua energia, uma terapêutica que pode proporcionar resultados positivos no bem-estar físico e psicológico e na consciência corporal de mulheres idosas participantes deum grupo que recebeu intervenção com esse recurso. 
Complementando os resultados encontrados neste estudo, Matos et al. (2018) apontam que a inclusão das PICs nos componentes curriculares de cursos como o de Enfermagem pode ser uma estratégia na formação e na capacitação desses profissionais, dando suporte e uma assistência cada vez mais voltada à integralidade do seu público.

\section{Conclusão}

Os achados deste estudo demonstraram que a utilização das práticas integrativas e complementares (PICs) proporciona impactos positivos a nível físico, emocional e espiritual. Observando a condição biológica e social da mulher e, ainda, que esta passa por diferentes contextos de vida, se justifica uma assistência em saúde com uma visão holística, integral, menos intervencionista e medicamentosa. Desse modo, as PICs se mostraram uma opção viável na promoção da saúde da mulher. Entretanto, faz-se necessário que a formação do profissional de saúdecontemple também este modelo assistencial.

Observa-se que, por questões culturais, o público feminino demonstra grande facilidade em aderir aos tratamentos alternativos e estão mais propensas a serem submetidas a intervenções com medicina integrativa, fator positivo para apesquisa e acompanhamento dos efeitos desta na promoção da saúde desse grupo.

Como limitação do presente estudo, ressalta-se a escassez de pesquisas que fazem comparação de grupos de mulheres que utilizam puramente a medicina convencional com grupos de mulheres que fazem uso de práticas integrativas e complementares atuando em conjunto com o tratamento convencional na promoção da saúde da mulher.

\section{Referências}

Banazeski, A. C., Luzardo, A. R., Rozo, A. J., Sinski, K. C., Palombit, M. R., \& Conceição, V. M. (2021). Percepções de enfermeiros sobre a atenção ao climatério. Revista de Enfermagem UFPE, 15(1), e245748. https://doi.org/10.5205/1981-8963.2021.245748

Bocanegra, B. M. P., Sosa, J. C. G; \& Simbaqueba, D. C. M. (2020). Terapias complementarias durante la gestación y parto. Revisión integrativa. Revista Cuidarte, 11(2), e1056. https://doi.org/10.15649/cuidarte.1056

Brasil. (2006). Ministério da Saúde. Portaria n. 971, de 03 de maio de 2006. Aprova aPolítica Nacional de Práticas Integrativas e Complementares (PNPIC) no Sistema Único de Saúde. Diário Oficial da União, Brasília, DF, maio de 2006.

Buglione, A., Saccone, G., Mas, M., Raffone, A., Di Meglio, L., Di Meglio, L., Toscano, P., Travaglino, A., Zapparella, R., Duval, M., Zullo, F., \& Locci, M. (2020). Effect of music on labor and delivery in nulliparous singleton pregnancies: a randomized clinical trial. Arch Gynecol Obstet, 301(3), 693-698. https://doi.org/10.1007/s00404-020-05475-9

Cicek S. \& Basar F. (2017). The effects of breathing techniques training on the duration of labor and anxiety levels of pregnant women, Complementary Therapies in Clinical Practice, 29 (1), 213-219. https://doi.org/10.1016/j.ctcp.2017.10.006

Coordenação Nacional de Práticas Integrativas e Complementares em Saúde. (2020). Contexto histórico da institucionalização das práticas integrativas e complementares em saúde no SUS. Guia de práticas integrativas e complementares em saúde para os gestores dos SUS. Dezembro, $2020,16 \mathrm{p}$, livreto 1.

Fancourt, D., \& Perkins, R. (2018). Could listening to music during pregnancy be protective against postnatal depression and poor wellbeing post birth? Longitudinalassociations from a preliminary prospective cohort study. BMJ Open, 8(7), e021251. http://dx.doi.org/10.1136/bmjopen-2017-021251

Fernández, F-X. A, Pino-Juste, M., \& Pérez, J. J. N. (2020). Beneficios de un programagrupal de tratamiento no farmacológico en el afrontamiento del cáncer en mujeres españolas. Una síntesis cualitativa. Saúde e Sociedade, São Paulo, 29(3), e181001. https://doi.org/10.1590/S0104-12902020181001

Governo do Estado do Maranhão. (2020). Maranhão é destaque pelo crescimento de unidades da Atenção Primária que ofertam Práticas Integrativas e Complementares. https://www.ma.gov.br/agenciadenoticias/?p=264053

Kao, Y-H., Huang, Y-C., Chung, U-L., Hsu, W-N., Tang, Y-T., \& Liao, Y-H. (2017). Comparisons for effectiveness of aromatherapy and acupressuremassage on quality of life in career women: a randomized controlled Trial. The Journal of Alternative and Complementary Medicine, 23(6), 451-460. http://doi.org/10.1089/acm.2016.0403

Kashani, L., Esalatmanesh, S., Eftekhari, F., Salimi, S., Foroughifar, T., Etesam, F., Safiaghdam, H., Moazen-Zadeh, E., Akhondzadeh. (2018). Efficacy of Crocus sativus (saffron) in treatment of major depressive disorder associated with post-menopausal hot flashes: a double-blind, randomized, placebocontrolled trial. Archives Gynecology and Obstetrics, 297(3), 717-724. https://doi.org/10.1007/s00404-018-4655-2

Kirshbaum, M. N, Stead, M, \& Bartys, S. (2016). An exploratory study of reiki experiences in women who have cancer. International Journal of Palliative Nursing, 22(4), 166-172. https://doi.org/10.12968/ijpn.2016.22.4.166

Martins, E. S., Rocha, L. M. A., De Jesus, A. P., Tavares, T. M., C., L., Castro, R. C. M. B., \& Pinheiro, A., K., B. (2020). Efeito da acupuntura para alívio dos desconfortos físicos eemocionais na gestação. Revista Fundamental Care, 12(1), 227-232. https://doi.org/10.9789/2175-5361.rpcfo.v12.8263 
Martins, E. S., Tavares, T. M. C. L., Lessa, P. R. A., Aquino, P. S., Castro, R. C. M. B., Pinheiro, A. K. B. (2018). Tratamento com acupuntura: avaliação multidimensional da dor lombar em gestantes. Revista da Escola de Enfermagem da USP, São Paulo, 52(1), e03323. https://doi.org/10.1590/S1980$220 \times 2017040303323$

Martins, E. S., Castro, R. C. M. B., Rocha, L. M. A., Pinheiro, A. K. B. (2017). Acupuntura e gravidez: medicina oriental como aliada no tratamento de dor lombar. Rev Rene, 18(4), 551-558. https://doi.org/10.15253/2175-6783.2017000400018

Matos, P. C., Laverde, C. R., Martins, P. G., De Souza, J. M., De Oliveira, N. F., \& Pilger, C. (2018). Práticas integrativas complementares na atenção primária àsaúde. Cogitare Enfermagem, Curitiba, 23(2), e54781. http://dx.doi.org/10.5380/ce.v23i2.54781

Melnyk, B. M., \& Fineout-Overholt, E (2005). Making the case for evidence based practice. In: $\quad$ Evidence based practice in nursing and healthcare: a guide to best practice. Philadelphia: Lippincot Williams \& Wilkins, 2005. p. 3-24.

Motta, P. M. R., \& Marchiori, R. A. (2013). Racionalidades médicas e práticas integrativas em saúde: estudos teóricos e empíricos. Cad. Saúde Pública, 29(4), 834-835. https://doi.org/10.1590/S0102-311X2013000400022

Organización Mundial de la Salud. (2020). Estrategia de la OMS sobre medicina tradicional 2014-2023. Ginebra: OMS, 2013. 75p. https://apps.who.int/iris/bitstream/handle/10665/95008/9789243506098_spa.pdf;jsessionid=5A830D0E242378A3615F5AEF79041D6B?sequence=1

Ozcan, H., Colak, P., Oturgan, B., \& Gülsever, E. (2019). Complementary and alternative treatment methods for menopausal hot flashes used in Turkey. African Health Sciences, 19(4), 3001-3008. https://doi.org/10.4314/ahs.v19i4.21

Rodriguez, M. P. C., Pacheco, S. C. C., Barbosa, M. R., C., S., S., \& Fernandes, J. L. L. (2018). A análise bioenergética como ferramenta de bem-estarnum grupo de mulheres idosas. Investigación en Enfermería: imagen y desarrollo, 20(2), 1-12. https://www.redalyc.org/journal/1452/145256681012/html/

Rosen, R. K., Thind, H., Jennings, E., Guthrie, K. M., Williams, D. M., \& Bock, B. C. (2016). "smoking does not go with yoga:" a qualitative study of women's phenomenological perceptions during yoga and smoking cessation. International Journal of Yoga Therapy, 26(1), 33-41. https://doi.org/10.17761/1531-2054-26.1.33

Santos, C. M. C., Pimenta, C. A. M., \& Nobre, M. R. C. (2007). A estratégia PICO para a construção da pergunta de pesquisa e busca de evidências. Revista Latino-Americana de Enfermagem, Ribeirão Preto, 15(3), 508-511. https://doi.org/10.1590/S0104-11692007000300023

Santos, V. R., \& Santos, K. O. B. (2017). Fisioterapia e práticas integrativas e complementares nos núcleos de apoio à saúde da família. Revista Pesquisa em Fisioterapia, 7(2), 207-214. http://dx.doi.org/10.17267/2238-2704rpf.v7i2.1318

Secretaria de Estado de Saúde de Minas Gerais. (2020). Práticas Integrativas e Complementares em Saúde. https://www.saude.mg.gov.br/pics

Da Silva, R. M., Jorge, H. M. F., Matsue, R. Y., Ferreira Junior, A. R., \& De Barros, N. F. (2016). Uso de práticas integrativas e complementares por doulas emmaternidades de Fortaleza (CE) e Campinas (SP). Saúde e Sociedade, São Paulo,25(1), 108-120. https://doi.org/10.1590/S0104-12902016143402

Styles, A., Loftus, V., Nicolson, S., \& Harms, L. (2019). Prenatal yoga for young women a mixed methods study of acceptability and benefits. BMC Pregnancy Childbirth, 19(1), 449. https://doi.org/10.1186/s12884-019-2564-4

Telesi Junior, E. (2016). Práticas integrativas e complementares em saúde, uma nova eficácia para o SUS. Estudos Avançados, São Paulo, 30(86), 99-112. https://doi.org/10.1590/S0103-40142016.00100007

Toneti, B. F., Avelar, J. M. P., Sousa, F. H., Toneti, A. N., Sonobe, H. M., \& Sawada, N. O. (2019). O significado de uma terapia integrativa de relaxamento guiado para mulheres com câncer de mama. Revista da Escola de Enfermagem da USP,53, e03947. http://dx.doi.org/10.1590/S1980- 220X2018024103497

Tsai, S-Y. (2016). Effect of Yoga Exercise on Premenstrual Symptoms among Female Employees in Taiwan. International Journal of Environmental Research andPublic Health, 13(7), 721. https://doi.org/10.3390/ijerph13070721

Ursi, E. S, \& Galvão, C. M. (2006). Prevenção de lesões de pele no perioperatório: revisãointegrativa da literatura. Revista Latino-Americana de Enfermagem, Ribeirão Preto, 14(1), 124-131. https://doi.org/10.1590/S0104-11692006000100017

Wang, Z., Dong, H., Wang, Q., Zhang, L., Wu, X., Zhou, Z-M., Yang, L., \& Huang, D. (2019). Effects of electroacupuncture on anxiety and depression in unmarried patients with polycystic ovarian syndrome: secondary analysis of a pilot randomised controlled trial. Acupuncture in Medicine, 37(1), 40-46. https://doi.org/10.1136/acupmed-2017-011615 